\title{
ANALYSIS OF CLINICAL RELATIONSHIP OF VISUAL ACUITY WITH OPTICAL COHERENCE TOMOGRAPHY AND PERIMETRY PARAMETERS IN PRIMARY OPEN-ANGLE GLAUCOMA
}

\author{
Mustafa Ömer İzzettinoğlu ${ }^{1}$ (D), Vuslat Gürlü ${ }^{2}$ (D)
}

${ }^{1}$ Trakya University School of Medicine, Edirne, TURKEY

${ }^{2}$ Department of Ophthalmology, Trakya University School of Medicine, Edirne, TURKEY

\begin{abstract}
Aims: This study aims to analyze the clinical relationship of visual acuity with optical coherence tomography and perimetry in primary open-angle glaucoma. Methods: This retrospective cross-sectional study was conducted with patients who were diagnosed with primary open-angle glaucoma in the ophthalmology department of Trakya University School of Medicine between January 2010 and January 2021. Perimetric data of patients such as visual field index, mean defect, pattern standard deviation, short-term fluctuation, corrected pattern standard deviation; results of optical coherence tomography such as average retinal nerve fiber layer thickness, average C/D ratio, and thickness of retinal nerve fiber layer quadrants, and records of examinational findings were statistically tested to evaluate the statistical relationship. Numbers, percentages, mean and standard deviation were used as the descriptive statistics. Results: Initially 80 eyes of 49 patients diagnosed with primary open-angle glaucoma met the inclusion criteria. 22 patients were female and 27 patients were male. Thinner mean retinal nerve fiber layer, inferior and superior quadrants were observed in eyes with lower visual acuity. It was observed that eyes with lower visual acuity had a worse visual field index and mean defect. Conclusion: In tertiary clinics that receive various numbers of patient referrals, perimetry can be seen as more useful and accurate in primary open-angle glaucoma detection and monitoring. It provides us with better and more accurate results for glaucoma management in the later stages. It is recommended that both optical coherence tomography and perimetry be used for disease monitoring, as this allows doctors to better monitor disease progression. In addition, since optical coherence tomography is an objective test and is less likely to require a patient response, it should be kept in mind that advanced and severe glaucoma may occur when performing perimetry, especially in patients with poor cooperation. Keywords: Glaucoma, perimetry, optical coherence tomography, visual field, epidemiology
\end{abstract}

\section{INTRODUCTION}

Primary open-angle glaucoma (POAG) is a chronic, degenerative optic neuropathy that causes selective loss of retinal ganglion cells, and is one of the leading causes of blindness worldwide. Even though POAG often occurs with elevated intraocular pressure (IOP), many POAG cases were reported to progress with a normal range of IOP values $(1,2)$. The condition of retinal ganglion cell loss leads to progressive enlargement of the optic nerve cup and thinning of the neural rim (3). Glaucoma is the leading cause of blindness, seen in 60 million people around the world (3). According to the survey data of 2013, POAG accounts for 44 million people across the world and is estimated to hit 53 million people by the year 2020 (4). According to recent studies in the United Kingdom, POAG is reported to be the most common type of glaucoma (5). Studies suggest that POAG is the second most common cause of blindness in the United States, approximately affecting 2.5 million people (6).

Optic disc findings frequently are detected before the deficit of the visual field appears. An enlarged cup-to-disc (C/D) ratio greater than $0.5, \mathrm{C} / \mathrm{D}$ ratio asymmetry of 0.2 or more between two eyes or a high asymmetry of the cup in one eye are considered to be diagnostic findings. The destructions caused by glaucoma are clinically revealed by OCT and perimetry tests (6). Perimetry, also known as vi- sual field testing, is a crucial diagnostic tool that maps out the visual field of the patient which makes it necessary for the diagnosis and management of POAG (7). Typical visual field changes in POAG include asymmetry of visual field between two eyes, temporal wedge, paracentral scotoma, general constriction of peripheral field, and increased variability of responses in an area which later developed field defects (7). The perimetry threshold for the detection of visual loss is estimated to reliably stand at $40-50 \%$ ganglion cell loss. Thus, in POAG, typical optic nerve head structural changes occur earlier than functional change (visual field loss) (7). It should be noted that there is the phase of characteristic glaucomatous changes and increased tendency of damages to the retinal nerve fiber layer (RNFL) that comes before the perimetrical defects are detectable (7).

This retrospective study aims to analyze the clinical relationship of best-corrected visual acuity (BCVA) with optical coherence tomography and perimetry on patients with POAG in the ophthalmology department of Trakya University School of Medicine.

\section{MATERIAL AND METHODS}

This study was approved by the Scientific Research Ethics Committee of Trakya University School of Medicine (Protocol Code: TÜTF-BAEK 2021/104). This retrospective cross-sectional study analyzed 80 eyes of 49 patients who were diagnosed with POAG 
in the Ophthalmology Department of Trakya University Hospital between January 2010 and January 2021. The study was carried out under the tenets of the Declaration of Helsinki. Written informed consent was obtained from all the participants.

Demographic data such as age and gender, accompanying systemic comorbidities such as hypertension and diabetes, risk factors such as high IOP levels, and the duration of onset POAG, the usage of topical prostaglandins, beta-blockers, carbonic anhydrase inhibitors and alpha agonists, initial and following clinical findings were obtained from the medical records of the patients. All patients underwent complete ophthalmologic examination at each visit including BCVA determined by the Snellen chart, anterior segment biomicroscopic examination, IOP measurement with Goldmann applanation tonometer, and detailed fundus examination obtained with 78 diopters non-contact lens.

The following findings were documented at the clinical visits: visual field parameters such as VFI, mean deviation, pattern standard deviation, optical coherence tomography (OCT) results such as mean RNFL thickness, mean C/D ratio, thicknesses of RNFL quadrants, surgical procedures that the patients underwent, the usage of topical anti-glaucomatous agents and types of anti-glaucomatous agents that have been used.

Initially, 80 eyes of 49 patients diagnosed with POAG met the inclusion criteria of having BCVA with positive light perception and above, absence of corneal pathologies, ocular-vascular diseases (central retinal artery occlusion, retinal venous occlusion), accompanying macular pathologies such as a macular hole and macular degeneration. POAG patients who have poorer BCVA than positive light perception, onset corneal pathologies, ocular-vascular diseases, and macular pathologies which could cause unreliable fundus examination results were excluded from this cross-sectional study.

Perimetric data of patients such as visual field index (VFI), mean defect (MD), pattern standard deviation (PSD), short-term fluctuation (SF), corrected pattern standard deviation (CPSD); results of OCT such as mean RNFL thickness, mean C/D ratio, and thickness of RNFL quadrants, and records of examinational findings were statistically tested to evaluate the statistical relationship.

Numbers, percentages, mean and standard deviation were used as the descriptive statistics. The variables were tested for normal distribution by the One-sample Kolmogorov-Smirnov test. The Chi-square test was used for qualitative comparison. Quantitative data were compared with the Independent Sample t-Test. The bivariate Pearson Correlation test was performed to measure the strength and direction of linear relationships between pairs of continuous variables. A p-value of $<0.05$ was set for statistical significance. The data were analyzed with IBM SPSS version 20.

\section{RESULTS}

In our study, 22 (44.9\%) patients were female and 27 (55.1\%) patients were male. The summary of patients' characteristics (mean age, duration of onset glaucoma, gender, accompanying comorbidity, and the number of the right or left eye that was affected) is shown in Table 1.

The mean BCVA for all eyes was $0.86 \pm 0.26$ Snellen decimals (ranging from 0.0080 to 1.0 Snellen decimals). It was found that bigger $\mathrm{C} / \mathrm{D}$ ratios due to fundus examination led to eyes in worsened BCVA ( $\mathrm{p}=0.018, \mathrm{r}=-0.263$, Pearson Correlation Test). Mean IOP was observed to be $16.3 \pm 3.5 \mathrm{~mm} \mathrm{Hg}$ (ranging from 6 to 23 $\mathrm{mm} \mathrm{Hg}$ ) and was not observed to have a noticeable correlation with BCVA $(\mathrm{p}=0.228)$. The summary of examinational findings (BCVA,
Table 1: Patient characteristics.

\begin{tabular}{lc} 
& Number of Patients [n (\%)] \\
\hline Age (years) & $63.1 \pm 10.2(43-84)$ \\
Duration of onset glaucoma (months) & $55.5 \pm 45.3(1-204)$ \\
Gender & \\
Female & $22(44.9)$ \\
Male & $27(55.1)$ \\
Affected eye & \\
Right & $40(50.0)$ \\
Left & $40(50.0)$ \\
Comorbidity & \\
Hypertension & $15(18.8)$ \\
Diabetes Mellitus & $12(16.3)$ \\
Hyperthyroidism & $2(2.5)$ \\
None & $49(59.9)$ \\
\hline
\end{tabular}

${ }^{*}$ Data were expressed as mean \pm SD (min-max).

Table 2: Examinational findings.

\begin{tabular}{lc} 
& Number of Patients [n (\%)] \\
\hline BCVA (Snellen Unit) ${ }^{*}$ & $0.86 \pm 0.26(0.0080-1.0)$ \\
IOP $(\mathbf{m m ~ H g})^{*}$ & $16.3 \pm 3.5(6-23)$ \\
Biomicroscopic findings & \\
Cataract & $3(3.9)$ \\
Nepheline & $2(2.5)$ \\
Nevus Filament & $1(1.3)$ \\
Pterygium & $2(2.5)$ \\
Biomicroscopic C/D ratio & \\
Lens statement & $0.6 \pm 0.3(0.1-1.0)$ \\
Phakic & \\
Pseudophakic & $60(75)$ \\
\hline
\end{tabular}

BCVA: Best corrected visual acuity, IOP: Intraocular pressure, C/D: Cup-to-disc

${ }^{*}$ Data were expressed as mean \pm standard deviation (min-max).

IOP, biomicroscopic findings, lens status, and biomicroscopic C/D ratio) was shown in Table 2.

In terms of perimetry and OCT results, the mean VFI was revealed as $74.2 \pm 34.6 \%$ (ranging from 0 to $100 \%$ ), and the mean thickness of RNFL was found out to be $76.9 \pm 17.5 \mu \mathrm{m}$. The summary of perimetry and OCT results (VFI, MD, PSD, SF, CPSD, mean $\mathrm{C} / \mathrm{D}$ ratio, mean RNFL thickness) is shown in Table 3.

When mean $\mathrm{C} / \mathrm{D}$ ratio and other quadrants of RNFL were observed, mean $\mathrm{C} / \mathrm{D}$ ratio, nasal and temporal quadrants did not have a significant correlation ( $\mathrm{p}=0.082$ for mean $\mathrm{C} / \mathrm{D}$ ratio, $\mathrm{p}=0.237$ for nasal quadrant, $\mathrm{p}=0.211$ for temporal quadrant). The inferior quadrant thickness had a negative correlation with age $(\mathrm{p}=0.01, \mathrm{r}=-$ $0.273)$. 
The mean thickness of the RNFL and BCVA had a statistically significant linear relationship $(\mathrm{p}<0.001, \mathrm{r}=0.418)$. Thinner RNFL was also observed in eyes with lower BCVA, indicating that they are positively correlated. VFI and BCVA also had a statistically significant linear relationship ( $\mathrm{p}=0.001, \mathrm{r}=0.441)$. It was observed that eyes with lower BCVA had worsened VFI. Additionally, MD had a statistically significant linear relationship with BCVA $(p<0.001$, $\mathrm{r}=0.545)$. RNFL inferior quadrant $(\mathrm{p}<0.001, \mathrm{r}=0.457)$ and RNFL superior quadrant $(\mathrm{p}=0.006, \mathrm{r}=0.303)$ had a moderate positive correlation with BCVA. All of the correlations are presented in Table 4 and Table 5.

The percentages of anti-glaucomatous agents' usage were observed to be $67.5 \%$ for prostaglandins, $68.8 \%$ for beta-blockers, $61.3 \%$ for carbonic anhydrase inhibitors, and $47.5 \%$ for alpha agonists. Regarding the operative approach, 11 (13.8\%) eyes underwent trabeculectomy, 8 (10.0\%) eyes underwent phacoemulsification cataract extraction and $1(1.3 \%)$ eye underwent vitrectomy.
Table 3: Perimetry and optical coherence tomography results.

Mean $\pm S D($ min-max $)$

\begin{tabular}{lc}
\hline Perimetry & $74.2 \pm 34.6(0-100)$ \\
VFI (\%) & $-9.6 \pm 9.1(-30.9-1.1)$ \\
MD (dB) & $5.6 \pm 3.1(1.5-14.4)$ \\
PSD (dB) & $2.0 \pm 1.0(0-5.7)$ \\
SF (dB) & $4.8 \pm 3.3(0-14.1)$ \\
CPSD (dB) & \\
Optical Coherence Tomography & $0.7 \pm 0.2(0.1-1.1)$ \\
Mean C/D Ratio & $76.9 \pm 17.5(40-115)$ \\
Mean RNFL Thickness $(\mu \mathrm{m})$ &
\end{tabular}

VFI: Visual field index, MD: Mean defect, PSD: Pattern standard deviation, SF: Short-term fluctuation, CPSD: Corrected pattern standard deviation, C/D: Cup-to-disc, RNFL: Retinal nerve fiber layer

Table 4: The strength and direction of linear relationships between pairs of continuous variables.

\begin{tabular}{|c|c|c|c|c|c|c|c|c|c|c|c|c|}
\hline \multirow[b]{2}{*}{ Continuous variables } & \multicolumn{2}{|c|}{ Age } & \multicolumn{2}{|c|}{$\begin{array}{l}\text { Duration of } \\
\quad \text { POAG }\end{array}$} & \multirow{2}{*}{$\begin{array}{c}\begin{array}{c}B B \\
\text { usage }\end{array} \\
\mathrm{z} \\
\end{array}$} & \multicolumn{3}{|c|}{ CAI usage } & \multicolumn{2}{|c|}{$\begin{array}{l}\text { Prostaglandin } \\
\text { usage }\end{array}$} & \multicolumn{2}{|c|}{ AA usage } \\
\hline & $\mathrm{r}$ & P-value & $\mathrm{r}$ & P-value & & P-value & $\mathrm{z}$ & P-value & $\mathrm{z}$ & P-value & $\mathrm{z}$ & P-value \\
\hline VFI & -0.141 & 0.213 & 0.084 & 0.0460 & -0.526 & 0.599 & -1.510 & 0.131 & -0.927 & 0.354 & -2.531 & 0.011 \\
\hline$M D$ & -0.165 & 0.144 & 0.117 & 0.300 & -0.706 & 0.480 & -1.639 & 0.101 & -0.801 & 0.423 & -2.895 & 0.004 \\
\hline$P S D$ & -0.118 & 0.299 & -0.002 & 0.98 & -0.140 & 0.889 & -0.523 & 0.601 & -1.998 & 0.046 & -1.600 & 0.110 \\
\hline The mean thickness of RNFL & -0.176 & 0.118 & 0.157 & 0.165 & -0.706 & 0.480 & -0.835 & 0.404 & -1.937 & 0.053 & -2.008 & 0.003 \\
\hline I Quadrant & -0.273 & 0.014 & 0.044 & 0.696 & -0.457 & 0.648 & -0.790 & 0.429 & -1.264 & 0.206 & -2.082 & 0.037 \\
\hline S Quadrant & -0.122 & 0.282 & 0.110 & 0.333 & -0.280 & 0.779 & -0.741 & 0.131 & -1.408 & 0.159 & -3.011 & 0.003 \\
\hline NQuadrant & -0.185 & 0.101 & -0.048 & 0.672 & -0.336 & 0.736 & -0.702 & 0.483 & -1.347 & 0.178 & -1.572 & 0.116 \\
\hline T Quadrant & -0.207 & 0.066 & -0.040 & 0.725 & -0.519 & 0.480 & -0.672 & 0.502 & -0.457 & 0.647 & -0.800 & 0.424 \\
\hline Mean C/D Ratio & -0.135 & 0.233 & 0.113 & 0.319 & -1.288 & 0.198 & -1.655 & 0.098 & -0.915 & 0.360 & -4.583 & $<0.001$ \\
\hline
\end{tabular}

VFI: Visual field index, MD: Mean defect, PSD: Pattern standard deviation, RNFL: Retinal nerve fiber layer, I Quadrant: Inferior quadrant, S Quadrant: Superior quadrant, $\mathbf{N}$ quadrant: Nasal quadrant, T Quadrant: Temporal quadrant, BB: Beta blocker, AA: Alpha agonist, CAI: Carbonic anhydrase inhibitor

Table 5: The strength and direction of linear relationships between pairs of continuous variables.

\begin{tabular}{lcc} 
& \multicolumn{2}{c}{ BCVA } \\
\cline { 2 - 3 } Continuous variables & $\mathrm{r}$ & $\mathrm{P}$-value \\
\hline VFI & 0.441 & 0.001 \\
MD & 0.545 & $<0.001$ \\
The mean thickness of RNFL & 0.418 & $<0.001$ \\
I Quadrant & 0.547 & $<0.001$ \\
S Quadrant & 0.303 & 0.006 \\
N Quadrant & 134 & 0.237 \\
T Quadrant & 0.141 & 0.211 \\
Mean C/D Ratio & -0.196 & 0.082 \\
Biomicroscopic C/D Ratio & -0.263 & 0.018 \\
IOP & 0.136 & 0.228 \\
\hline
\end{tabular}

BCVA: Best-corrected visual acuity, $\boldsymbol{r}$ : Pearson correlation coefficient, VFI: Visual field index, MD: Mean defect, RNFL: Retinal nerve fiber layer, I quadrant: Inferior quadrant, S quadrant: Superior quadrant, $N$ quadrant: Nasal quadrant, T quadrant: Temporal quadrant, C/D: Cup-to-disc, IOP: Intraocular pressure

\section{DISCUSSION}

Primary open-angle glaucoma is an asymptomatic, progressive, and degenerative optic neuropathy characterized by enlarging optic disc and loss of visual field. Patients at high risk for POAG include black people older than 40 years, white people older than 65 years, and people with a family history of glaucoma or people with a history of diabetes or severe myopia (-6.00 diopters or more, i.e., farther from 0.00) (6). In our study, the mean age of people with POAG was 63.1 years, many other studies confirming an older age prevalence as well $(1,6,8-10)$. It was also seen that hypertension and diabetes were two of the major risk factors for POAG. Other studies with different ethnicities have revealed that blood pressure changes and onset diabetes played a major role in increasing the risk of POAG $(11,12)$. Therefore, adequate management of blood pressure, blood sugar levels, and HbAlc are crucial for decreasing the risk of POAG.

In our study, mean BCVA results were seen as 0.86 , with mean IOP being in the normal range. Our study did not reveal a statistically significant linear relationship between IOP and BCVA. The fact that POAG could occur and progress with normal IOP should not be overlooked. The study with 229 eyes conducted by Omodaka et al. (13) confirmed that IOP had a weak correlation with BCVA, 
which is in line with the results of our study. According to the study of Leung et al. (14), better BCVA is more correlated with normal RNFL thickness, especially with the thickness of the temporal quadrant RNFL. On the contrary, our study revealed the temporal quadrant of RNFL to not have a correlation with BCVA, unlike superior and inferior quadrants which have strong correlations with BCVA. This situation could be explained by the earlier and more destructive effects of POAG on inferior and superior quadrants. In addition, it was seen that older patients had thinner inferior RNFL quadrant. It is believed to occur due to the age-related changes in the retinal layer of the globe. Moreover, the mean thickness of the RNFL was within the normal range in our study, which could explain the reason for the better BCVA results.

Visual field loss is also one of the other causes of POAG. Peripheral vision loss is usually the initial defect and is followed by central vision loss (blindness) in POAG. Perimetry should be done in patients with POAG to detect visual field defects. Perimetry is the gold standard investigation tool in which defects are detectable when $40-50 \%$ loss of ganglion cells occurs. Therefore, it is believed that structural tests can identify progression in the early stages better while perimetry is more useful in the later stages (15-20). Thus, it is believed that perimetry is more relevant in the later stages of POAG (18). On the contrary, OCT detects even the slightest defects on the optic disc head which gives a higher detection chance of disease progression in the early stages and is still indeed a handy tool for POAG and examination of worsened BCVA. Nonetheless, the fact that moderate BCVA could be gotten despite OCT results even with minimal defects should be noted (7). Therefore, examination of peripapillary RNFL thickness will provide a better chance of detecting early glaucoma than that of perimetry $(14,18)$. In our study, it was observed that perimetry parameters were more accurate in POAG than the results of OCT in which VFI and MD had better strength to reveal the defects than the parameters of OCT such as mean RNFL thickness, superior and inferior quadrants of RNFL. These results could be attributed to the fact that the patient group in our study was referred to our tertiary clinic from another one. Additionally, the study of Banegas et al. (16) supported the functionality of OCT in progression detection of pre-perimetric glaucoma, while perimetry is more reliable in later stages. Given these facts, we believe that worsened BCVA is better explained with perimetry in the tertiary clinics.

The main limitation of our study is that our clinic is considered a tertiary center which meant that the majority of our patients were already on anti-glaucomatous medications at the time of their referral, indicating that their POAG was relatively under control. This left us with better parameters of POAG such as BCVA, IOP, RNFL thickness, C/D ratio, which were used for investigation and management.

In conclusion, OCT has higher sensitivity in the detection of progression than perimetry in the early stages, which gives OCT the ability of progression detection within a shorter follow-up time in early POAG. On the other hand, perimetry provides us better and more accurate results for POAG management in the later stages. Therefore, our study has revealed that in tertiary clinics that receive various numbers of patient referrals, perimetry is more useful and accurate in POAG detection and monitoring. Using both OCT and perimetry for disease monitoring is advisable, as this provides us better disease progression tracking with a better chance than using one method without the other.
Ethics Committee Approval: This study was approved by the Scientific Research Ethics Committee of Trakya University School of Medicine (Protocol Code: TÜTF-BAEK 2021/104).

Informed Consent: Informed consent was obtained from the participants of this study.

Conflict of Interest: The authors declared no conflict of interest.

Author Contributions: Concept: MÖİ, VG. Design: MÖİ, VG. Supervision: MÖİ, VG. Resources: MÖİ, VG. Materials: MÖİ, VG. Data collection and/ or processing: MÖİ, VG. Analysis and/or Interpretation: MÖİ, VG. Literature Search: MÖİ, VG. Writing Manuscript: MÖİ, VG. Critical Review: MÖİ, VG.

Financial Disclosure: The authors declared that this study received no financial support.

\section{REFERENCES}

1. Kwon YH, Fingert JH, Kuehn MH et al. Primary open-angle glaucoma. N Engl J Med 2009;360(11):1113-24.

2. Singh LN, Crowston JG, Lopez Sanchez MIG et al. Mitochondrial DNA variation and disease susceptibility in primary open-angle glaucoma. Invest Ophthalmol Vis Sci 2018;59(11):4598-602.

3. Şimşek T. Current diagnosis and management of primary angle closure glaucoma. Osmangazi Journal of Medicine 2018;40(2):90-100.

4. Kapetanakis VV, Chan MP, Foster PJ et al. Global variations and time trends in the prevalence of primary open angle glaucoma (POAG): a systematic review and meta-analysis. Br J Ophthalmol 2016;100(1):86-93.

5. Kroese M, Burton H. Primary open angle glaucoma. The need for a consensus case definition. J Epidemiol Community Health 2003;57(9):752-4.

6. Distelhorst JS, Hughes GM. Open-angle glaucoma. Am Fam Physician 2003;67(9):1937-44

7. Mahabadi N, Foris LA, Tripathy K. Open angle glaucoma. (cited 2020 Nov 20) Treasure Island (FL): StatPearls Publishing; 2021.

8. Leske MC, Connell AM, Schachat AP et al. The Barbados Eye Study. Prevalence of open angle glaucoma. Arch Ophthalmol 1994;112(6):821-9.

9. Wormald RP, Basauri E, Wright LA et al. The African Caribbean Eye Survey: risk factors for glaucoma in a sample of African Caribbean people living in London. Eye (Lond) 1994;8:315-20.

10. Jiang X, Varma R, Wu S et al. Baseline risk factors that predict the development of open-angle glaucoma in a population: the Los Angeles Latino Eye Study. Ophthalmology 2012;119(11):2245-53.

11. Zhao D, Cho J, Kim MH et al. Diabetes, fasting glucose, and the risk of glaucoma: a meta-analysis. Ophthalmology 2015;122(1):72-8.

12. Zhou M, Wang W, Huang W et al. Diabetes mellitus as a risk factor for open-angle glaucoma: a systematic review and meta-analysis. PLoS One 2014;9(8):102972.

13. Omodaka K, Nakazawa T, Yokoyama Y et al. Correlation between peripapillary macular fiber layer thickness and visual acuity in patients with open-angle glaucoma. Clin Ophthalmol 2010;4:629-35.

14. Leung CK, Chan WM, Yung WH et al. Comparison of macular and peripapillary measurements for the detection of glaucoma: an optical coherence tomography study. Ophthalmology 2005;112(3):391-400.

15. Hood DC, Kardon RH. A framework for comparing structural and functional measures of glaucomatous damage. Prog Retin Eye Res 2007;26(6):688-710.

16. Banegas SA, Anton A, Morilla A et al. Evaluation of the retinal nerve fiber layer thickness, the mean deviation, and the visual field index in progressive glaucoma. J Glaucoma 2016;25(3):229-35.

17. Sommer A, Katz J, Quigley HA et al. Clinically detectable nerve fiber atrophy precedes the onset of glaucomatous field loss. Arch Ophthalmol 1991;109(1):77-83.

18. Abe RY, Diniz-Filho A, Zangwill LM et al. The relative odds of progressing by structural and functional tests in glaucoma. Invest Ophthalmol Vis Sci 2016;57(9):421-8

19. Ohnell H, Heijl A, Brenner L et al. Structural and functional progression in the early manifest glaucoma trial. Ophthalmology 2016;123(6):1173-80.

20. Zhang X, Dastiridou A, Francis BA et al. Advanced imaging for glaucoma study group. Comparison of glaucoma progression detection by optical coherence tomography and visual field. Am J Ophthalmol 2017;184:63-74. 